\title{
EURECA - The Future of Cryogenic Dark Matter Detection in Europe
}

\begin{abstract}
H Kraus $^{1 \star}$, E Armengaud ${ }^{7}$, M Bauer ${ }^{4}$, I Bavykina ${ }^{2}$, A Benoit ${ }^{13}$, A Bento ${ }^{2}$, $\mathrm{J}_{\text {Blümer }}{ }^{56}$, L Bornschein ${ }^{5}$, A Broniatowski ${ }^{10}$, G Burghart ${ }^{9}, \mathrm{P}$ Camus ${ }^{13}$, A Chantelauze ${ }^{6}$, M Chapellier $^{8}$, G Chardin ${ }^{10}$, C Ciemniak $^{3}$, C Coppi $^{3}, \mathrm{~N} \mathrm{Coron}^{12}$, O Crauste ${ }^{10}$, F A Danevich ${ }^{17}$, M De Jésus ${ }^{11}$, P de Marcillac ${ }^{12}$, E Daw ${ }^{16}$, X Defay ${ }^{10}$, G Deuter ${ }^{4}$, J Domange ${ }^{7}$, P Di Stefano ${ }^{11}$, G Drexlin ${ }^{5}$, L Dumoulin ${ }^{10}$, $\mathrm{K}_{\text {Eitel }}{ }^{6}$, F von Feilitzsch ${ }^{3}$, D Filosofov ${ }^{14}$, P Gandit ${ }^{13}$, E Garcia ${ }^{15}$, J Gascon ${ }^{11}$, G Gerbier ${ }^{7}$, J Gironnet ${ }^{12}$, H Godfrin ${ }^{13}$, S Grohmann ${ }^{6}$, M Gros ${ }^{7}$, M Hannewald ${ }^{7}$,

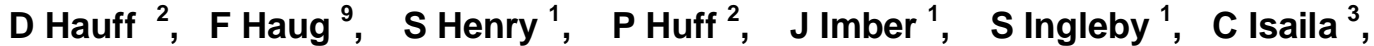
$\mathrm{J}$ Jochum $^{4}$, A Juillard ${ }^{10}$, M Kiefer ${ }^{2}$, M Kimmerle ${ }^{4}$, H Kluck ${ }^{6}$, V V Kobychev ${ }^{17}$, V Kozlov ${ }^{6}$, V A Kudryavtsev ${ }^{16}$, T Lachenmaier ${ }^{3}$, J-C Lanfranchi ${ }^{3}$, R F Lang ${ }^{2}$, P Loaiza ${ }^{18}, \quad$ A Lubashevsky ${ }^{14}, \quad$ M Malek ${ }^{1}, \quad$ S Marnieros ${ }^{10}, \quad$ R McGowan ${ }^{1}$, V Mikhailik ${ }^{1}$, V M Mokina ${ }^{17}, \quad$ A Monfardini $^{13}, \quad$ X-F Navick ${ }^{7}, \quad$ T Niinikoski $^{9}$, A S Nikolaiko ${ }^{17}$, L Oberauer $^{3}$, E Olivieri ${ }^{10}, \mathrm{Y} \mathrm{Ortigoza}^{15}$, E Pantic ${ }^{2}$, P Pari ${ }^{8}$, B Paul $^{7}$, G Perinic ${ }^{9}$, F Petricca ${ }^{2}$, S Pfister ${ }^{3}$, C Pobes ${ }^{15}$, D V Poda ${ }^{17}$, R B Podviyanuk ${ }^{17}$, O G Polischuk ${ }^{17}$, W Potzel ${ }^{3}$, F Pröbst ${ }^{2}$, J Puimedon ${ }^{15}$, M Robinson ${ }^{16}$, S Roth ${ }^{3}$, K Rottler ${ }^{4}$, S Rozov ${ }^{14}$, C Sailer ${ }^{4}$, A Salinas ${ }^{15}$, V Sanglard ${ }^{11}, \mathrm{M}$ L Sarsa ${ }^{15}, \mathrm{~K}$ Schäffner ${ }^{2}$, S Scholl ${ }^{4}$, S Scorza ${ }^{11}$, A Smolnikov ${ }^{14}$, W Seidel ${ }^{2}$, S Semikh ${ }^{14}$, M Stern ${ }^{11}$, L Stodolsky ${ }^{2}$, M Teshima ${ }^{2}$, V Tomasello ${ }^{16}$, A Torrento ${ }^{9}$, L Torres ${ }^{15}$, V I Tretyak ${ }^{17}$, J A Villar ${ }^{15}$, M A Verdier ${ }^{11}$, I Usherov ${ }^{4}$, $\mathrm{J}$ Wolf $^{5}$, E Yakushev ${ }^{14}$
\end{abstract}

1 University of Oxford, Department of Physics, Keble Road, Oxford OX1 3RH, UK;

2 Max-Planck-Insitut für Physik, Föhringer Ring 6, 80805 Munich, Germany;

3 Technische Universität München, Physik Department E15, 85748 Garching, Germany;

4 Eberhard Karls Universität Tübingen, Auf der Morgenstelle 14, 72076 Tübingen, Germany;

5 Institut für Experimentelle Kernphysik, Universität Karlsruhe (TH), Gaedestrasse 1, 76128 Karlsruhe, Germany;

6 Forschungszentrum Karlsruhe, Institut für Kernphysik, Postfach 3640, 76021 Karlsruhe, Germany;

7 CEA, Centre d'Etudes Saclay, IRFU, 91191 Gif-Sur-Yvette Cedex, France;

8 CEA, Centre d'Etudes Saclay, IRAMIS, 91191Gif-Sur-Yvette Cedex, France;

9 CERN, 1211 Geneva 23, Switzerland;

10 CSNSM, Université Paris-Sud and CNRS/IN2P3, 91405 Orsay, France;

11 Université de Lyon, F-69622, Lyon, France; Université de Lyon 1, Villeurbanne; CNRS/IN2P3,

Institut de Physique Nucléaire de Lyon; 
12 Institut d'Astrophysique Spatiale, UMR-8617 CNRS / Univ Paris Sud, Bat. 12191405 Orsay Cedex, France;

13 CNRS-Neel, 25 Avenue des Martyrs, 38042 Grenoble cédex 9, France;

14 Joint Institute for Nuclear Research, DLNP, 141980 Dubna, Moscow Region, Russia;

15 Laboratorio de Fisica Nuclear y Astropartículas, Facultad de Ciencias, Universidad de Zaragoza, C/

Pedro Cerbuna 12, 50009 Zaragoza, Spain;

16 Department of Physics and Astronomy, The University of Sheffield, Hicks Building, Hounsfield Road, Sheffield, S3 7RH, UK;

17 Institute for Nuclear Research, MSP 03680 Kyiv, Ukraine;

18 Laboratoire Souterrain de Modane, 90, Rue Polset, 73500 Modane, France

E-mail: h.krausephysics.ox.ac.uk

The European Underground Rare Event Calorimeter Array (EURECA) will be an astro-particle physics facility in the Laboratoire Souterrain de Modane, aiming to directly detect galactic dark matter. The EURECA collaboration unites CRESST, EDELWEISS and the Spanish-French experiment ROSEBUD, thus concentrating and focussing effort on cryogenic detector research in Europe into a single facility. The aim is to explore WIMP - nucleon scalar cross sections in the $10^{-9}-10^{-10}$ picobarn region with a target mass of up to one ton. A major advantage of EURECA is the planned use of more than just one target material (multi target experiment for WIMP identification).

Identification of dark matter 2008

Stockholm, Sweden

August 18-22, 2008 


\section{Motivation}

Recent experimental data on the cosmic microwave background, combined with other astronomical and astrophysical data, have significantly improved the precision of the fundamental parameters in our cosmological model [1]. Much of the matter density of the Universe seems to comprise non-luminous, non-baryonic particles [2]. Supersymmetry provides weakly interacting massive particles (WIMPs) as appealing and well-motivated candidates for this dark matter [3]. The WIMP-nucleon cross section is at or below the electroweak scale and the expected event rates are correspondingly low. The identification of a WIMP interaction in a detector is therefore challenging, owing to the rate of WIMP interactions being very small compared with the event rates expected from background radioactivity of present detectors with highest purity and from cosmic radiation. In addition, the recoil energies produced by elastic WIMP-nucleus scattering are very small, in the range of a few $\mathrm{keV}$ to a few tens of keV.

To address these experimental challenges, a new generation of cryogenic detectors has been developed, exhibiting powerful background discrimination in combination with unprecedented energy threshold and resolution [4 -6]. The detectors allow highly efficient identification of nuclear recoils (caused by WIMP and also neutron interaction) by eliminating electron recoils due to radioactivity. Their use in the existing EDELWEISS-II and CRESST-II dark matter searches is providing valuable R\&D, expertise and experience for EURECA.

EURECA aims to having a target sensitivity a factor $>100$ better than projected by current phase II experiments. Although it is not unlikely that a discovery will be made at WIMPnucleon cross sections above $10^{-8}$ picobarn, the range between $10^{-8}$ and $10^{-10}$ picobarn is currently most favoured [7] and will be covered by EURECA. At the lower end of cross section values, this translates to only a few events per ton per year in typical targets, requiring ultra-low background environments and excellent event type discrimination, neutron moderators and muon vetos.

\section{Cryogenic detector technology}

EURECA's detectors are based on those used in the European CRESST, EDELWEISS and ROSEBUD experiments. The detectors are low-temperature calorimeters, operating in the millikelvin temperature range, using complementary techniques for discrimination of nuclear and electron recoil events. Detectors based on charge-phonon detection [4] are used by EDELWEISS. The thermal signal induced by energy deposition in a germanium detector crystal is measured with a high-impedance thermistor attached to its surface. Simultaneously, the ionization signal is read out via electrodes on the crystal surface. The ratio between the measured ionisation and heat signals provides an efficient tool for the identification of the event type. Detectors based on scintillation-phonon [5] are used by CRESST and ROSEBUD. Many materials scintillate and CRESST has currently $\mathrm{CaWO}_{4}$ absorbers and one $\mathrm{ZnWO}_{4}$ crystal installed. The thermal signal is measured with a superconducting transition edge sensor (TES) on the crystal surface. Simultaneously, scintillation is detected with thin calorimeters also using a TES sensor, but optimized for detection of scintillation. 
The aim of EURECA R\&D is to explore concepts and designs, based on the existing technologies, appropriate to a large-scale experiment. The exploitation phases of EDELWEISSII and CRESST-II are aligned with the R\&D for and design of EURECA. This should allow selecting the optimum detector technology for EURECA. An important aspect in designing a ton-scale experiment is the inherent scalability of cryogenic detectors. Individual modules have already been developed and optimised; scaling up merely requires the production of more modules and a larger cold space in a dilution refrigerator. Increasing the production rate of modules does not require additional detector development, although further improvement is always possible and likely to occur as a by-product of optimising the manufacturing process. Use of a larger cryostat does not affect the operation of individual detectors. Large dilution refrigerators are in use already, for example for gravitational wave antennae, but cooling large detector arrays to $\sim 10$ millikelvin with several thousand wires attached represents a challenge.

A modular approach is vital to achieving large detector masses. There are likely to be limitations in large-scale detectors due to radioactive backgrounds present in the target materials. With individual sub-kilogram solid targets, modules with abnormally high backgrounds can be isolated and replaced. Fitting individual readout channels to each target mass also helps keep the data stream free from pile-up and makes calibration and monitoring easier.

An important feature of EURECA will be its multi-material target. Having several targets is highly desirable for testing the correct A-scaling of WIMP-nucleon interactions and to determine residual neutron backgrounds, if present. Further strong motivation for equipping EURECA with a range of target materials is provided by kinematic considerations, as the mass of the WIMP is unknown. A natural initial choice for EURECA is to use germanium and tungstate targets, given the expertise of the collaboration. Additional absorbers are being researched and optimized [8-11].

Arranging the detectors in a large array of smaller absorbers has the further advantage of allowing testing for uniform rates within the target, and for providing an additional dark matter signature by requiring single interactions only for a dark matter candidate event. This should allow identification of residual neutron background through coincidences.

\section{EURECA - Next Steps}

\subsection{Cryogenics and Shielding}

The design of the cryogenic and shielding infrastructure requires detailed studies and careful planning. EURECA will require significant improvements in the radiopurity of the immediate environment to achieve the very low background rate required. This concerns gamma ray background and neutron-induced background. The depth of the underground site is a key factor for achieving lowest muon-induced backgrounds, and therefore, EURECA will be installed within the Laboratoire Souterrain de Modane (LSM). The EURECA collaboration is working closely with the LSM on the design of dedicated integrated shielding and accommodation of cryogenic equipment in the planned extension of the LSM. 


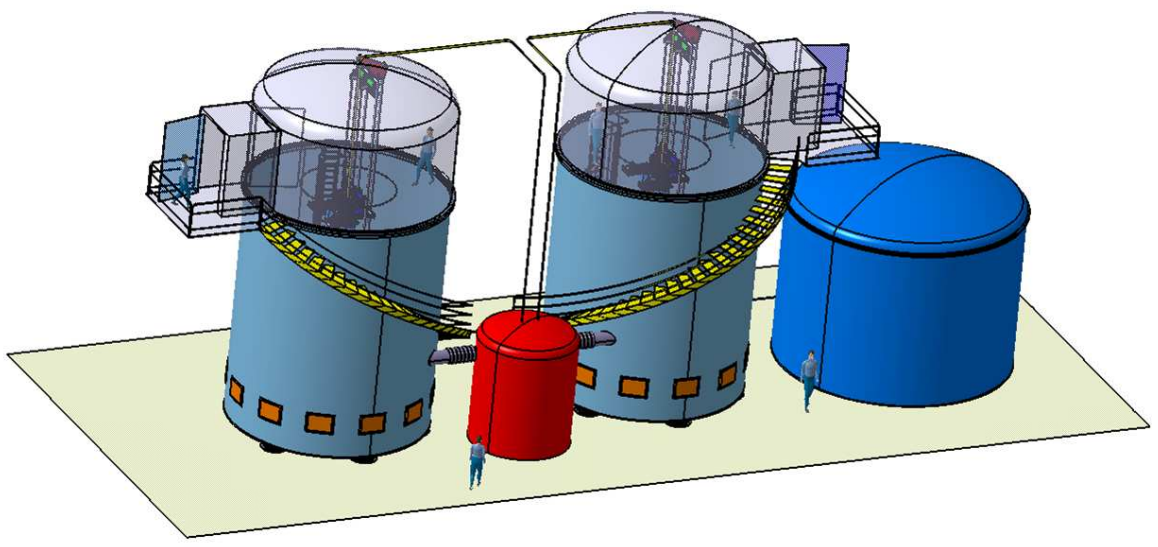

Fig. 1: A possible EURECA facility design, currently under study by the collaboration

An early artist's view of the EURECA setup with water shields around the two cryostats is shown in Fig. 1. Dilution refrigeration will be used to cool the ton-scale cold mass of EURECA. Studies are under way to determine the cooling power required of the dilution refrigerator of EURECA. First results indicate a considerable heat load, which is in good agreement with experimental values obtained for large cold masses of similar size in other experiments. The proximity cryogenics must satisfy the stringent requirements resulting from remote operation in underground laboratories. In addition, much has been learned from the rather different design concepts of CRESST and EDELWEISS. The lessons learned from the operation of these experiments will have their impact on the design of the EURECA cryogenic system.

\subsection{Electronics}

Electronic readout and DAQ will have to scale to several thousand channels. Some of the scalability issues have already been addressed in providing the readout hardware for the present experiments, but a further $\sim 10$-fold increase in density, reduction of heat loads through readout wires, and a reduction in the cost per channel will be necessary for the realization of EURECA.

In order to reduce the cost per readout channel to an acceptable level we will need to develop multi-channel front-end electronics, to replace the present single-channel systems; and redesign detector bias and control systems to take advantage of the economy of scale. Some hardware components can be avoided by providing their function through software.

Multiplexing systems for cryogenic detectors have been developed for astronomical arrays to reduce the number of readout channels. It is not yet clear if these can be adapted for a dark matter search, but it is likely there will be some sort of multiplexing at some level.

Digitization at an early stage in the DAQ chain will be crucial. This approach reduces cost and adds flexibility. Rather than building trigger hardware, it is foreseen to use arrays of field programmable gate arrays (FPGA) in which triggers, arranged by their level of sophistication, can be realized. Research and development has started already, with "intelligent" electronics, capable of identifying potentially interesting signals above noise. A trigger system with several 
levels allows further balancing the available computing power between the levels of sophistication of the trigger condition, successively reducing the volume of the data stream as it propagates through the data pipeline.

With the number of readout channels increasing, automated control and monitoring becomes increasingly important. We will need to further develop the capability of operating the experiment from outside the underground laboratory, and to keep track of an increasing number of parameters in order to certify the origin of any event in our detector.

A larger experiment means longer cables and a greater number of passages through thermal shields and heat sinks. This is less a problem for low-impedance detector readout channels, but might cause problems for high-impedance read-out due to stray and parasitic capacitance. To somewhat alleviate these potential drawbacks, research will be necessary into specific grounding schemes, possibly involving actively driven shields and a comparison of various possible implementation, such as coax cables or strip lines. Avoiding cross talk between channels despite the high density of cables is also an important issue.

The readout system and DAQ will need to be able to accommodate a variable number of detectors with very different design, operation and speed. We aim to design it as modular as possible so that a large number of sections / components will be the same across the range of readout configurations. Other aspects to include are linking the output from the muon veto into the data acquisition system and ensuring synchronisation of time stamp recording with precision at the microsecond level.

Many of the above ideas have already passed their first stage of research and development and are currently implemented in EDELWEISS-II and CRESST-II. The combination of expertise and experience gained in these two experiments is being harnessed for the next level of scaling up.

\subsection{Detectors}

A major task faced by EURECA will be mass production of the detector modules, moving away from prototyping, which, by its nature, has a low rate of detector production. An important aspect is the screening of raw detector materials before they enter the production process. This has to be done to a level compatible with sensitivities of measuring only a few events per year.

A key feature of EURECA is its operation of different target materials and complementary discrimination technologies within a single common volume. Here we combine the advantages of the detector concepts, namely scintillation and heat (as in CRESST and ROSEBUD), and ionization and heat (as in EDELWEISS). The long-term aim is rationalization of the detector sensors and streamlining the corresponding readout chains. The heat signal could be measured using either high-impedance thermistors or transition edge sensors. Gluing of thermometers (rather than fabricating them directly on surface of the dark matter target) separates thermometer optimization from choosing a specific absorber material [12 - 14].

Regarding the supply of scintillating absorbers in large quantities, we are working with suppliers on the reduction of radioactive impurities and at the same time aim to improve the light yield and size of the scintillators. In this systematic approach we put great emphasis on 
reproducibility, reliability and quality control. An investigation into the possibility of growing our own crystals is also under way.

We will have to improve considerably the radiopurity of the bulk detector materials. To perform this, careful selection of materials is needed, which requires a large number of samples to be tested with sensitivities of at least two orders of magnitude better than the present ones. For EDELWEISS-II, this is currently done using a high-sensitivity low-background Ge gamma spectrometer on the basis of a HP Ge diode of $210 \mathrm{~cm}^{3}$ with archaeological lead shielding, situated in a deep underground facility. The best sensitivity obtained, for instance in measurements of copper samples with mass of about $10 \mathrm{~kg}$ for a measuring time of about 1 month is at the $\mathrm{mBq} / \mathrm{kg}$ level for U/Th and ${ }^{60} \mathrm{Co}$. This sensitivity limit could be improved, for example by reducing the background of the Ge spectrometers, by increasing the masses of the samples as well as the effective mass of the Ge detector, and by redesigning the geometry of the spectrometer, aiming for $4 \pi$ coverage.

The storage of materials, manufacturing of devices and growth of crystals underground would prevent cosmogenic activation. An adequate underground environment should consist of large storage areas and dedicated clean rooms, with sensitive radon monitoring and trapping facilities ( $\mathrm{Rn}$ activity $\sim \mathrm{mBq} / \mathrm{m}^{3}$ of air). While storage underground is not a huge problem for the small amount of material in the current experiments, it will need a dedicated effort for the increased space requirements of EURECA.

\section{References}

[1] D N Spergel et al., Astrophys. J. Suppl. 148 (2003) 175.

[2] L Bergström, Rep. Prog. Phys. 63 (2000) 793.

[3] B W L Lee and S Weinberg, Phys. Rev.Lett. 39 (1977) 165.

[4] D Sanglard et al., Phys. Rev. D 71 (2005) 122002.

[5] G Angloher et al., Astropart. Phys. 23 (2005) 325 and arXiv:0809.1829 (astro-ph).

[6] D S Akerib et al., Phys. Rev. Lett. 96 (2006) 011302.

[7] J Ellis et al., Phys. Rev. D 71 (2005) 095007.

[8] H Kraus et al., Phys. Lett. B 610 (2005) 37.

[9] V B Mikhailik and H Kraus, J. Phys. D: Appl. Phys. 39 (2006) 1181.

[10] F A Danevich et al., Phys. Stat. Sol. A 205 (2007) 335.

[11] I Bavykina et al., IEEE Transactions on Nuclear Science, 55 (2008) 1449.

[12] J-C Lanfranchi et al., Nucl. Instr. Meth. A 520 (2004) 135.

[13] S Roth et al., arxiv:0810.0423 (astro-ph), to be published in the proceedings of CryoScint08, Optical Materials, Elsevier.

[14] M Kiefer et al., arxiv:0809.4975(astro-ph), to be published in the proceedings of CryoScint08, Optical Materials, Elsevier. 